\title{
Creating Pseudocolored Images in Photoshop
}

Jerry Sedgewick

University of Minnesota sedge@umn.edu

The method for creating pseudocolor in Photoshop can be done in few steps, but the image will not be colored in the conventional way. The conventional means for producing pseudocolor comes by using a look up table (LUT). This LUT is an overlay for making a range of colors according to choices provided by the software. The colors are matched to levels of brightness and darkness in the image (grayscale pixel values), typically from a short to long wavelengths. In that scenario, violet colors are matched to the black and near-black pixels and red to those close to absolute white. Blue, yellow, green and orange become the mid-range pixel values.

The beauty of the LUT method for coloring images lies in the ability to see pseudocolor "live" while imaging. Also, many software programs provide a means for editing the range of colors to amplify differences in pixel values when shifts in pixel densities/intensities are subtle, or when researchers want to show differences at thresholds where colors change (a pseudocolor bar included with the image also needs to be edited in the same way and shown).

The bane lies both in the difficulty understanding how to make these changes in software programs and in the frustrating inability to save the pseudocolor image with the Save function. The latter doesn't make sense until one understands that the pseudocolor is really a temporary overlay: the computer is "looking up" the colors for immediate display on the monitor. Therefore, the pseudocolor does not exist as a change to the image itself.

You can capture these images, however, by simply holding down the control key on a desktop PC and by pressing the Print Screen button, often at the top, right of the keyboard. On a Macintosh, you can hold down the Apple, Shift and F3 key at the same time (at least in pre-OS $X$ operating systems). You will hear a sound like the click of a camera shutter to denote that your screen has been saved.

In both cases, the image is copied to random memory. You will have to open Photoshop, under File choose New, click Okay without changing any settings; and then, under Edit, choose Paste. An image of the entire screen will appear on your new file. Use the crop tool to surround the pseudocolored image and double click inside the cropped area to complete the crop. This image will contain as many pixels across and down as it did on the computer screen and that resolution depends on the settings for your monitor. Thus, the greater the settings for pixels across and down for the monitor, the greater the resolution will be in your copied image.

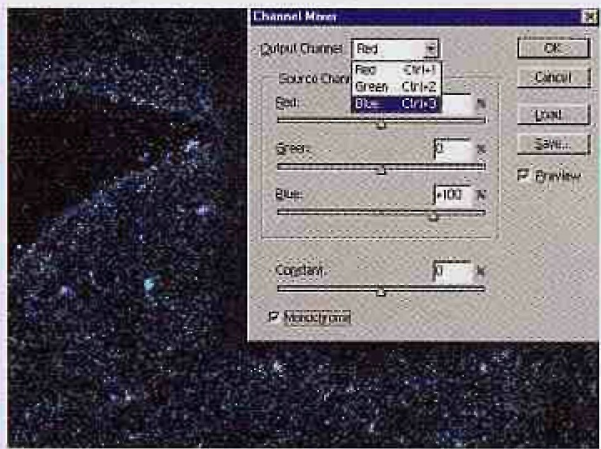

Figure 1: Use Channel Mixer when changing the mode of RGB color, fiuorescent images to grayscale. The image above was collected with blue illumination and saved as RGB color. Blue is selected as the Output Channel and the Monochrome box is checked to make the image into grayscale values.
If you would like, instead, to color these images in Photoshop, and to make changes to the pseudocolor (and to the image itself), you can do so by following these steps:

1. Make the grayscale image Indexed Color. Under Image on the menu, choose Mode then indexed Color. Indexed colored images contain 256 grayscale or color values, as many as contained in an 8-bit image.

This assumes that the image is grayscale. If the image is in color, then change the image to grayscale first. For variously colored images, such as those collected on a brightfield microscope, simply change the image to RGB: under Image on the menu, choose Mode then RGB Color. In this scenario, the green channel is chosen as the grayscale image, this most matches what we see by eye.

If the image contains shades of one color only (such as fluorescent images), then change to grayscale first by using the appropriate channel. Under Image, choose Mode then Channel Mixer. Select the Output Color that matches the color of your image (e.g., if your image is red, choose Red as the Output Color) and check the Monochrome box at the bottom, left of the dialogue box

Note: you may get a dialogue box indicating a "palette" recommended by Photoshop. It should read "Exact" and the forced output should be Black and White. In that manner, the grayscale values will be preserved. Other palettes adapt grayscale values for various outputs, such as the black and white values that best fit those of the web.

2. Choose pseudocolor table. Under Image on the menu, choose Mode and then Color Table to choose the pseudocolor table. The only available table that is typically used in biology would be Spectrum. If your image contains values from deep black to near white, then the colors may appear as desired.

If, however, the colors do not convincingly reveal desired regions of interest, then two choices follow: either change the image itself to fit the pseudocolor table, or change the pseudocolor table to fit the image. Most researchers would choose the latter, because the image is raw data and it should not be tampered with. If grayscale values are altered in the image, then only linear changes in histogram values can be made: the white and black sliders can be used in the Levels dialogue box. The gamma (middle) slider cannot be used because it results in logarithmic changes. Any changes to one image must also be made to other images included in the same figure.

Changes in either the image or in the pseudocolor table require the inclusion of a color bar so that any alterations can be done on both the image and the bar. Viewers can then make sense of these changes by looking at the bar 3. Make a color bar. Under Image on the menu, choose New and specify the height and width of the bar $(400 \times 30$ pixels works for me). Choose Grayscale for Mode. Near the bottom of the toolbar

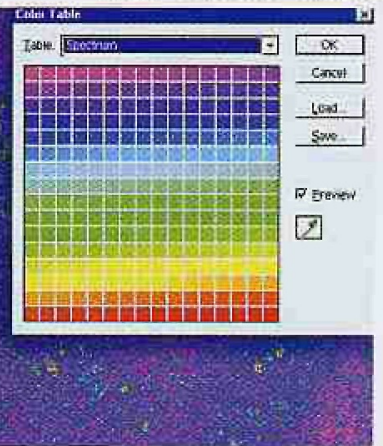

Figure 2: To pseudocolor the grayscale image, change the mode to Indexed Color and then choose Color Table in the Image > Mode menu. Select Spectrum for the Table.

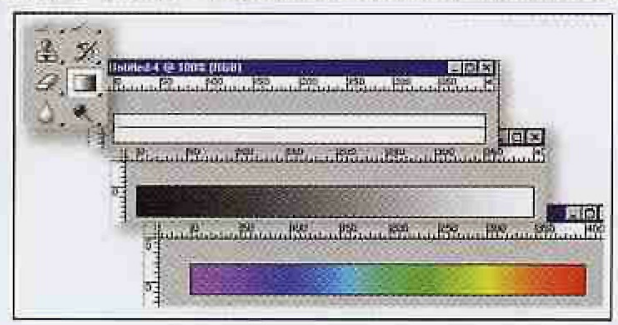

Figure 3: To make a pseudocolor bar, choose the gradient tool from the toolbar (top left), and drag the cursor from one end of a New file to the other while holding down the shift key. A grayscale gradient from black to white with all values between will be created. Change the Mode of the image to Indexed Color, then choose Color Table and Spectrum to pseudocolor gradient. 


\section{Simultaneous FIB Milling and High Resolution SEM Imaging}

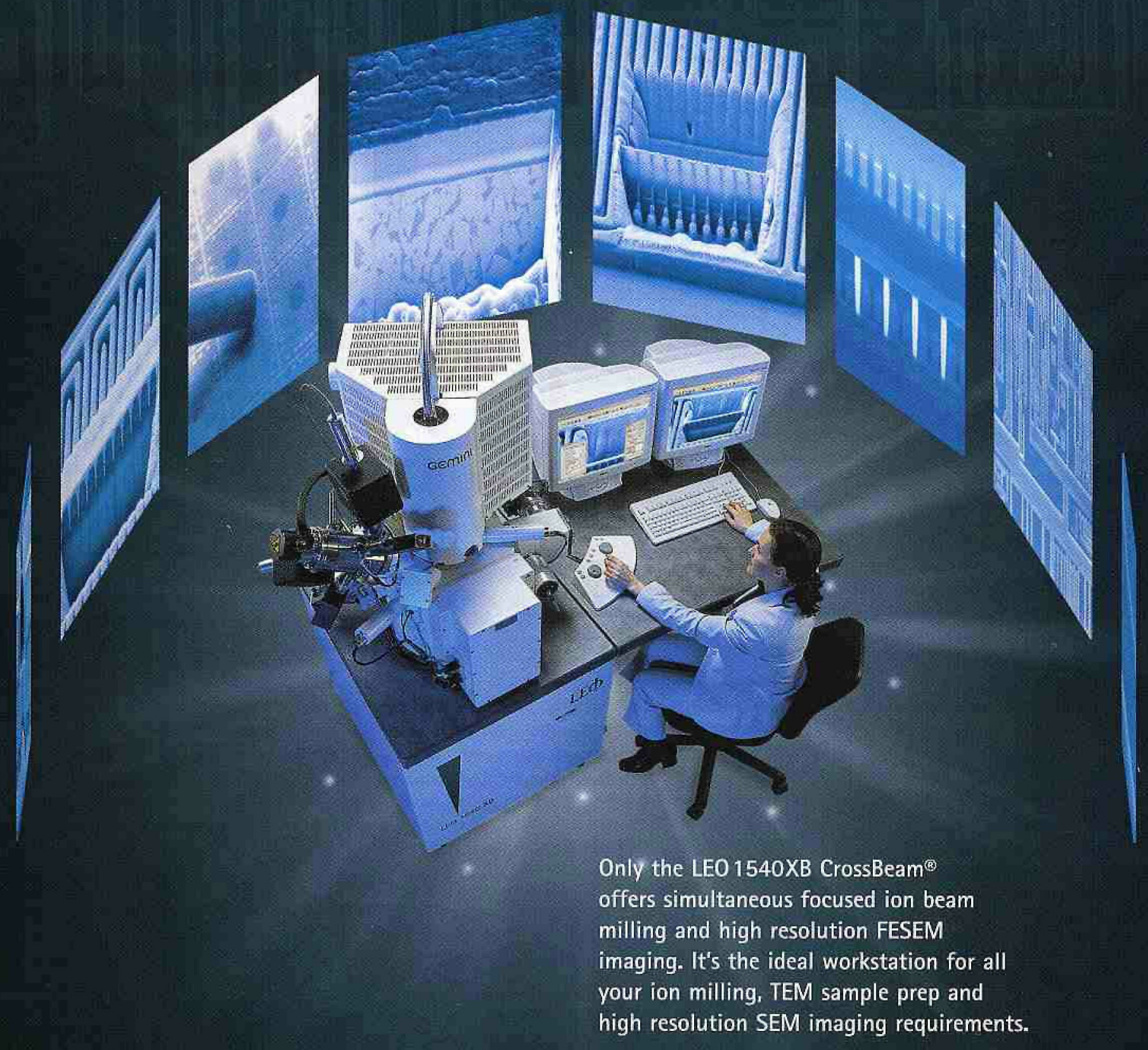

LEO Electron Microscopy Gioup A Carl Zeiss SMT AG Company 73417 Oberkochen, Germony TEl: $++497364-946137$

Fax: $++497364-944851$

info@leo.de - mwwleo-em.com
LEO Electron Microscopy Inc A Carl Zeiss SMT AG Company Thornwood, New York 10594 Toll Free: $800-356-1090$ Fax: $914-681-7443$

wurwleo-usa.com

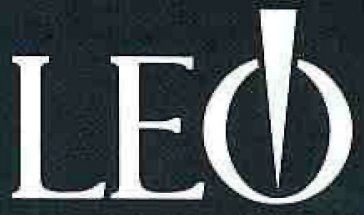




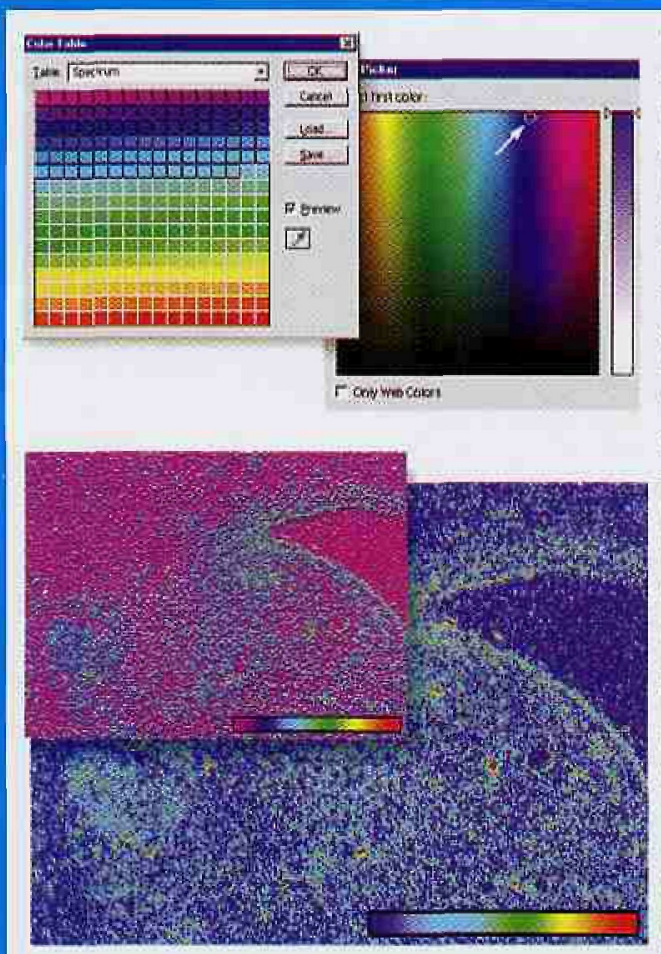

Figure 4: To change colors in the pseudocolored image, edit the Spectrum color table. For the images above, the violet color made the background visually confusing. To re-color the background, the cursor is dragged across color squares matched to darkest pixel values in the image (top left). The Select First Color dialogue box appears and blue color is selected from a color swatch by clicking on it (top right). Another dialogue box appears (Select Second Color) and a color close to cyan is selected from the color swatch (not shown). Bottom left image shows background colors before changes, bottom right image shows new background colors. while looking at values in the Info box. The black end should read 0 and the right 255 with all other values in fixed increments along the gradient.

Now make the grayscale into pseudocolor by changing the Mode to Indexed Color (under Image > Mode > Indexed Color). Choose Color Table under Image > Mode and choose the Spectrum table to make into ranges of colors. You may also want to label the bar to indicate where incremental grayscale values lie.

Make sure that you place the color bar on the same page as your image so that all changes to the color table will affect both.

Note that the colors are not fixed by equal increments: blue and green and red take up an inordinate amount of grayscale values compared to violet, cyan and yellow.

4. If desired, change the pseudocolor table. Changes in the table will require an Investment of time and trial and error. Perhaps the best way to begin would be to visually determine what values would be regarded as background on the image and then change those colors in the image and the pseudocolor bar. These values are often placed between violet and the blue just shy of cyan.

To alter the colors on the table, under /mage on the menu, choose Mode and then Color Table. Drag the cursor over the violet-to-blue or violet-to-cyan squares to highlight all of them. You will get a second dialogue box to indicate the first color (if you see Web Colors checked at the bottom of the First Color dialogue box, click to uncheck to make it easier to navigate the color spectrum). Click on violet color to choose it (or color of your choice), click Okay. Then you will get a second dialogue box asking you to choose the second color. Choose a blue near cyan or cyan (or another color of your choice), click Okay. The image will now show large areas of violet and blue showing background levels. Be sure to save the color table before exiting, because you will want to apply this table to subsequent images in a figure.

If you did not get what you desired, click cancel and start again, this time choosing different ranges of color squares in the Color Table dialogue box or choose different First and Second colors. Continue to re-color other grayscale levels by choosing colors of your choice in the same manner.

With a little work, this method should give you the color table you desire without the necessity for changing grayscale values on the image itself. Be sure to alter colors for publication photos when you are required to submit CMYK images to obtain good reproduction according to methods described in earlier editions of Microscopy Today.

\section{ELECTRON MICROSCOPIST} University of Wisconsin-Madison

The Materials Science Center at the University of Wisconsin invites applications for an electron microscopist to oversee its transmission electron microscopy facility. The facility includes high resolution and energy filtered imaging, STEM, EDS, EELS, and sample preparation. It supports a broad range of research by students, staff, and faculty with interests in materials characterization. The candidate's responsibilities will include user training and assistance, instrument maintenance, and instrument development.

Candidates must have a strong background in the theory and practice of TEM micro-characterization of materials with a minimum of 2 years post-graduate experience. The candidate should also have a strong background in vacuum technology, electronics, and computers. Strong interpersonal and communications skills are essential. Candidates should have an MS ( $\mathrm{PhD}$ preferred) in a physical science or engineering discipline. Candidates with a BS in physical science or engineering with professional experience and knowledge in the required field equivalent to an MS will also be considered. Candidates are encouraged to view additional information at http://www.ohr.wisc.edu/pvl/pv_045842.html.

Interested candidates should send a resume and cover letter referring to Position Vacancy Listing \#45842 to Richard Noll, 1509 University Ave., Madison, WI 53706, or email to noll@engr.wisc.edu.

To ensure full consideration, all information should be received by November 30,2003 . Note that unless confidentiality is requested in writing, information regarding the names of applicants must be released upon request. Finalists cannot be guaranteed confidentiality.

UW-Madison is an equal opportunity/ affirmative action employer. We promote excellence through diversity and encourage all qualified individuals to apply.
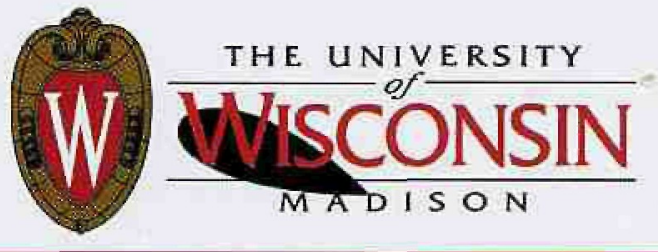

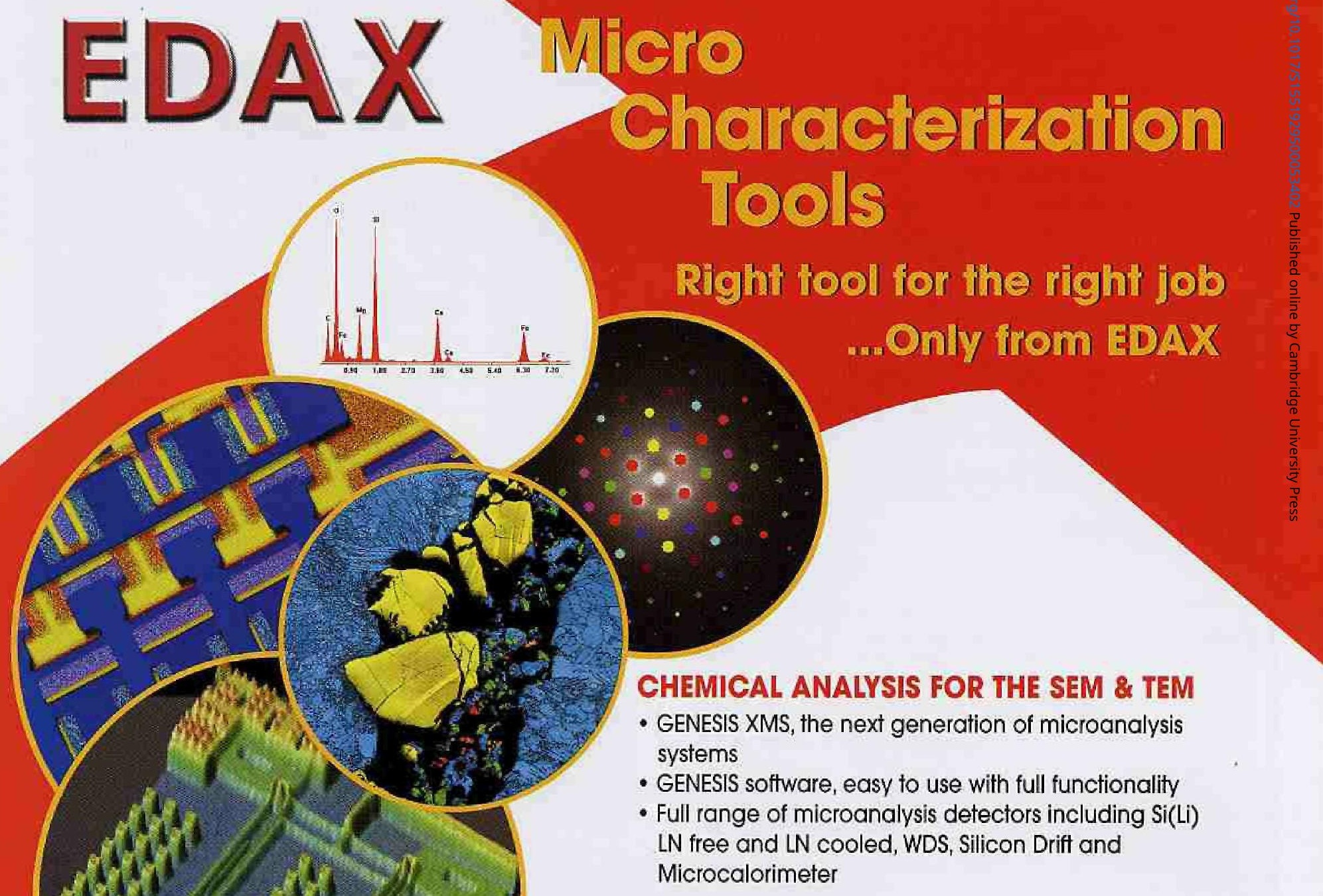

\section{STRUCTURAL ANALYSIS IN THE SEM \& TEM}

- EBSD in the SEM

OIM provides crystal orientation mapping and texture analysis

Delphi combines EBSD and EDS data for phase identification

- Electron Diffraction in the TEM

$\mathrm{ACT}$, automated tools for the acquisition, analysis and reporting of TEM diffraction data

\section{INTEGRATED SOLUTIONS}

- Take micro characterization to the next level and achieve the most accurate results with simultaneous EDS/EBSD data acquisition and Chl-Scan chemical indexing

\section{PHASE-CONTRAST IMAGING}

Americas $201-529-4880$
Jaban $+881-3-3740-5172$

- Reveal the internal microstructure of a sample with Europe, Africa and Middle East
$+31-13-536-4000$

S.E Asla +852-2698-7373 